through the orifice in the mesocarp so that the embryo can emerge. The caps are somewhat similar to those of Sclerocarya and Dracontomelon, but they are triangular in section and hollowed within to contain the upper part of the embryo. In shape they resemble a French forage cap.

The extreme case of wastage of effort is that of the Brazil nut, Bertholletia. When you purchase Brazil nuts, you rarely, if ever, see the fruit body in which they are contained. The well-known 'nuts' are the seeds with their strong, woody seedcoat; but they are contained in a large, woody, spherical fruit some six inches in diameter, with a wall half-an-inch thick and as hard as wellseasoned oak, with a smooth, glass-like inner layer. At one end of the ball there is a small orifice firmly plugged by a stopper, and inside the 15-20 seeds are so neatly packed, with their thin edges inwards, that the hollow wooden sphere is completely filled, and no space is wasted. When conditions are favourable for germination, the seeds inside all commence to germinate at once. The orifice, halfan-inch across, however, is their only means of escape, as the fruit wall remains hard and intact. The result may be compared with the rush of a crowd on the call of "Fire" at a theatre. Everyone tries to get out at once and only one out of the 15-20 prisoners survives! Surely this is a case where the means have defeated the end.

Tennyson may well have had the Brazil nut in mind when, referring to Nature, he wrote :

"So careful of the type she seems, So careless of the single life ;

\section{That I, considering everywhere}

Her secret meaning in her deeds,

And finding that of fifty seeds

She often brings but one to bear,

I falter where I firmly trod,"

Why should some seeds, like those of many orchids and lilies, papery in their texture and almost transparent, survive perfectly well in a dormant condition for a long period, while others need a strong protective envelope?

All these questions relating to the nature of the life in a dormant seed, whether germination may be immediate or may be long delayed, and the ingenious methods of germination, afford problems of much interest; all the more so since they are so illusive and because our attempts to solve them are confronted by so many difficulties.

\title{
Possible Production of Elements of Atomic Number Higher than 92
}

\author{
By Prof. E. Fermi, Royal University of Rome
}

$\mathrm{U}$ NTIL recently it was generally admitted that an atom resulting from artificial disintegration should normally correspond to a stable isotope. M. and Mme. Joliot first found evidence that it is not necessarily so ; in some cases the product atom may be radioactive with a measurable mean life, and go over to a stable form only after emission of a positron.

The number of elements which can be activated either by the impact of an $\alpha$-particle (Joliot) or a proton (Cockcroft, Gilbert, Walton) or a deuton (Crane, Lauritsen, Henderson, Livingston, Lawrence) is necessarily limited by the fact that only light elements can be disintegrated, owing to the Coulomb repulsion.

This limitation is not effective in the case of neutron bombardment. The high efficiency of these particles in producing disintegrations compensates fairly for the weakness of available neutron sources as compared with $\alpha$-particle or proton sources. As a matter of fact, it has been shown ${ }^{1}$ that a large number of elements (47 out of 68 examined until now) of any atomic weight could be activated, using neutron sources consisting of a small glass tube filled with beryllium powder and radon up to 800 millicuries. This source gives a yield of about one million neutrons per second.

All the elements activated by this method with intensity large enough for a magnetic analysis of the sign of the charge of the emitted particles were found to give out only negative electrons. This is theoretically understandable, as the absorption of the bombarding neutron produces an excess in the number of neutrons present inside the nucleus; a stable state is therefore reached generally through transformation of a neutron into a proton, which is connected to the emission of a $\beta$-particle.

In several cases it was possible to carry out a chemical separation of the $\beta$-active element, following the usual technique of adding to the irradiated substance small amounts of the neighbouring elements. These elements are then separated by chemical analysis and separately checked for the $\beta$-activity with a Geiger-Müller counter. The activity always followed completely a certain element, with which the active element could thus be identified.

In three cases (aluminium, chlorine, cobalt) the active element formed by bombarding the element of atomic number $Z$ has atomic number $Z-2$. In four cases (phosphorus, sulphur, iron, zine) the atomic number of the active product is $Z-1$. In two cases (bromine, iodine) the active element is an isotope of the bombarded element.

This evidence seems to show that three main processes are possible: (a) capture of a neutron with instantaneous emission of an $\alpha$-particle; (b) capture of the neutron with emission of a 
proton ; $(c)$ capture of the neutron with emission of a $\gamma$-quantum, to get rid of the surplus energy. From a theoretical point of view, the probability of processes $(a)$ and $(b)$ depends very largely on the energy of the emitted $\alpha$ - or H-particle; the more so the higher the atomic weight of the element. The probability of process $(c)$ can be evaluated only very roughly in the present state of nuclear theory; nevertheless, it would appear to be smaller than the observed value by a factor 100 or 1,000 .

It seemed worth while to direct particular attention to the heavy radioactive elements thorium and uranium, as the general instability of nuclei in this range of atomic weight might give rise to successive transformations. For this reason an investigation of these elements was undertaken by the writer in collaboration with F. Rasetti and O. D'Agostino.

Experiment showed that both elements, previously freed of ordinary active impurities, can be strongly activated by neutron bombardment. The initial induced activity corresponded in our experiments to about 1,000 impulses per minute in a Geiger counter made of aluminium foil of $0.2 \mathrm{~mm}$. thickness. The curves of decay of these activities show that the phenomenon is rather complex. A rough survey of thorium activity showed in this element at least two periods.

Better investigated is the case of uranium; the existence of periods of about 10 sec., 40 sec., 13 min., plus at least two more periods from 40 minutes to one day is well established. The large uncertainty in the decay curves due to the statistical fluctuations makes it very difficult to establish whether these periods represent successive or alternative processes of disintegration.

Attempts have been made to identify chemically the $\beta$-active element with the period of $13 \mathrm{~min}$. The general scheme of this research consisted in adding to the irradiated substance (uranium nitrate in concentrated solution, purified of its decay products) such an amount of an ordinary $\beta$-active element as to give some hundred impulses per minute on the counter. Should it be possible to prove that the induced activity, recognisable by its characteristic period, can be chemically separated from the added activity, it is reasonable to assume that the two activities are not due to isotopes.

The following reaction enables one to separate the $13 \mathrm{~min}$.-product from most of the heaviest elements. The irradiated uranium solution is diluted in 50 per cent nitric acid ; a small amount of a manganese salt is added and then the manganese is precipitated as dioxide $\left(\mathrm{MnO}_{2}\right)$ from the boiling solution by addition of sodium chlorate. The manganese dioxide precipitate carries a large percentage of the activity.

This reaction proves at once that the 13 min.activity is not isotopic with uranium. For testing the possibility that it might be due to an element 90 (thorium) or 91 (palladium), we repeated the reaction at least ten times, adding an amount of uranium $\mathbf{X}_{1}+\mathbf{X}_{2}$ corresponding to about 2,000 impulses per minute; also some cerium and lanthanum were added in order to sustain uranium $\mathrm{X}$. In these conditions the manganese reaction carried only the 13 min.-activity; no trace of the 2,000 impulses of uranium $\mathrm{X}_{1}$ (period 24 days) was found in the precipitate; and none of uranium $\mathbf{X}_{2}$, although the operation had been performed in less than two minutes from the precipitation of the manganese dioxide, so that several hundreds of impulses of uranium $\mathbf{X}_{2}$ (period 75 sec.) would have been easily recognisable.

Similar evidence was obtained for excluding atomic numbers 88 (radium) and 89 (actiniüm). For this, mesothorium-1 and -2 were used, adding barium and lanthanum; the evidence was completely negative, as in the former case. The eventual precipitation of uranium- $X_{1}$ and meso. thorium-1, which do not emit $\beta$-rays penetrating enough to be detectable in our counters, would have been revealed by the subsequent formation respectively of uranium- $\mathrm{X}_{2}$ and mesothorium-2.

Lastly, we added to the irradiated uranium solution some inactive lead and bismuth, and proved that the conditions of the manganese dioxide reaction could be regulated in such a way as to obtain the precipitation of manganese dioxide with the 13 min.-activitv without carrying down lead and bismuth.

In this way it appears that we have excluded the possibility that the 13 min.-activity is due to isotopes of uranium (92), palladium (91), thorium $(90)$, actinium (89), radium (88), bismuth (83), lead (82). Its behaviour excludes also ekacæsium (87) and emanation (86).

This negative evidence about the identity of the 13 min.-activity from a large number of heavy elements suggests the possibility that the atomic number of the element may be greater than 92 . If it were an element 93 , it would be chemically homologous with manganese and rhenium. This hypothesis is supported to some extent also by the observed fact that the 13 min.-activity is carried down by a precipitate of rhenium sul. phide insoluble in hydrochloric acid. However, as several elements are easily precipitated in this form, this evidence cannot be considered as very strong.

The possibility of an atomic number 94 or 95 is not easy to distinguish from the former, as the chemical properties are probably rather similar. Valuable information on the processes involved could be gathered by an investigation of the possible emission of heavy particles. A careful search for such heavy particles has not yet been carried out, as they require for their observation that the active product should be in the form of a very thin layer. It seems therefore at present premature to form any definite hypothesis on the chain of disintegrations involved.

1 E. Fermi, Ricerca Scientifica, 1, 5, $283 ; 6,330$. NATURE, 133, 757 May 19, 1934. E. Amaldi, O. D'Agostino, E. Fermi, F. Rasetti, E. Segre, Ricerca Scientifica, 8, 452, 1934 